\title{
High-dose Tamoxifen plus Ifosfamide and Anthracycline in a Patient with Angiosarcoma of the Breast
}

\author{
C-H Hsiao ${ }^{1,2}, \mathrm{~K}-\mathrm{H} \mathrm{Yeh}^{2,3}$, Y-C Chang ${ }^{4}$, C-C Tsai ${ }^{5}$
}

\begin{abstract}
Although breast cancer is, unfortunately, not uncommon in women, a mere $0.04 \%$ of malignant breast tumours are primary angiosarcomas. Chemotherapy is advocated for treatment of breast angiosarcomas; however, no guidelines exist regarding optimal chemotherapeutics or protocols. Presently, the prognosis for breast angiosarcomas is poor. This case report describes a 24-year old woman diagnosed with primary breast angiosarcoma. She initially refused to receive treatment, but later returned to the hospital four years later with a haemopneumothorax. She was treated with rescue chemotherapy using a combination of high-dose tamoxifen plus ifosfamide and epirubicin (an anthracycline). She achieved a partial response, but died 16 months after therapy was initiated. More research is needed to devise novel chemotherapeutics and protocols to improve outcomes in women diagnosed with primary angiosarcomas of the breast.
\end{abstract}

Keywords: Angioscarcoma, anthracycline, breast cancer, chemotherapy, ifosfamide, tamoxifen

\section{Tamoxifen en Dosis Alta con Ifosfamida y Antraciclina en un Paciente con Angiosarcoma de Mama

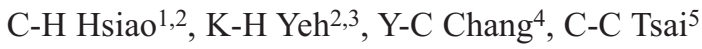

\begin{abstract}
RESUMEN
Aunque el cáncer de mama, desafortunadamente, no es poco común en las mujeres, apenas $0.04 \%$ de los tumores malignos de mama son angiosarcomas primarios. La quimioterapia es el tratamiento de preferencia en los casos de angiosarcomas de mama. Sin embargo, no existen guías en relación con los protocolos o la quimioterapia óptima. En la actualidad, el pronóstico para los angiosarcomas de mama es pobre. Este informe del caso describe a una mujer de 24 años diagnosticada con angiosarcoma primario de mama. Inicialmente la paciente se negó a recibir tratamiento, pero volvió al hospital cuatro años más tarde con un hemoneumotórax. Fue tratada entonces con quimioterapia de rescate usando una combinación de alta dosis de tamoxifen con ifosfamida y epirrubicina (antraciclina). Llegó a responder parcialmente al tratamiento, pero falleció 16 meses después del inicio de la terapia. Se necesitan más investigaciones para elaborar nuevos quimioterápeuticos y protocolos que mejoren los resultados en los casos de mujeres diagnosticadas con angiosarcomas primarios de mama.
\end{abstract}

Palabras claves: Angiosarcoma, antraciclina, cáncer de mama, quimioterapia, ifosfamida, tamoxifen

West Indian Med J 2013; 62 (7): 651

From: ${ }^{1}$ Division of Hematology-Oncology, Department of Internal Medicine, Far-Eastern Memorial Hospital, ${ }^{2}$ Cancer Research Center and ${ }^{3}$ Department of Oncology, College of Medicine, National Taiwan University, ${ }^{4}$ Department of Chest Surgery and ${ }^{5}$ Department of Pathology, Far-Eastern Memorial Hospital, Taiwan.
Correspondence: Dr C-H Hsiao, Division of Hematology-Oncology, Department of Internal Medicine, Far-Eastern Memorial Hospital, No 21 Section 2, Nan-Ya South Road, Ban-Chiao, New Taipei City, 222, Taiwan, ROC. Fax: +886-2-77282166, e-mail: ch_hsiao@icloud.com 


\section{INTRODUCTION}

Breast cancer in women is not an uncommon entity. Approximately one in eight women will be diagnosed with breast cancer (1). Less than $1 \%$ of breast malignancies are sarcomas, and a mere $0.04 \%$ of malignant breast tumours are primary angiosarcomas (2-4). Usually, breast cancer is less common in women aged 20-24 years than older women. In the case of angiosarcoma, however, younger women (30-50 years) are more typically affected (4). Because of the rarity of this condition, few reports and guidelines describing the management of this condition are published. Glazebrook et al reported that of an estimated 2500 breast biopsies examined between January 2004 and June 2006, only four cases of primary angiosarcoma were diagnosed (4). Similarly, Bae et al reported that only 9/9129 women were diagnosed with primary angiosarcoma of all breast malignancies diagnosed at the Samsung Medical Center in Seoul, Korea between 1997 and 2011 (2).

Unlike other forms of breast cancer, there are no firm treatment guidelines, such as those published by the National Comprehensive Cancer Network, for the diagnosis, treatment, and follow-up of patients diagnosed with primary angiosarcoma of the breast (5). This report describes a patient with advanced primary angiosarcoma treated with highdose tamoxifen plus ifosfamide and anthracycline.

\section{CASE REPORT}

A 24-year old woman identified a lump measuring approximately $3 \mathrm{~cm}$ in diameter in the lateral upper quadrant of her left breast during a self-examination in 2000. Following sonography and mammography, an excisional biopsy was performed by a breast surgeon at another medical centre. Pathology was supportive of angiosarcoma. Cells were arranged in anastomosing vascular channels with papillary projections. The vascular spaces were lined by atypical plump cells exhibiting pleomorphic nuclei and occasional mitotic figures with focally epithelioid appearance. These atypical cells were positive for CD31 and factor VIII but negative for cytokeratin (CK), oestrogen receptor (ER), progesterone receptor (PR), and human epidermal growth factor receptor (Her2/neu). After being informed of the biopsy results, the patient was reluctant to return to the hospital despite frequent telephone contact and was subsequently lost to follow-up. In July 2004, the patient presented to an emergency room at a local community hospital with acute respiratory distress and a haemopneumothorax. She was transferred to the author's medical centre after initial supportive measures. An emergent exploratory thoracotomy was performed and an $8 \mathrm{~cm}$ diameter tumour on the thoracic wall was identified, which had ruptured and was actively bleeding. Haemostasis was achieved by suture ligation and a biopsy revealed angiosarcoma. The same pattern of cancer cells in anastomosing vascular channels with papillary projections lined by atypical cells was noted. These tumour cells were also positive for factor VIII but negative for CK, ER, PR, and
Her2/neu. A more comprehensive physical examination performed postoperatively revealed a second painless lump in her right breast. Biopsy confirmed that this tumour was also an angiosarcoma, and computed tomography (CT) identified a central necrotic liquefied area with peripheral rim enhancement. The patient deteriorated rapidly. Systemic chemotherapy was initiated in August 2004. Specifically, the regimen consisted of tamoxifen $150 \mathrm{mg} / \mathrm{m}^{2} /$ day which was divided into four doses and administered daily for seven days. The tamoxifen was started three days before administering a continuous infusion of ifosfamide $2.5 \mathrm{~g} / \mathrm{m}^{2} /$ day for three days with mesna uroprotection and a concomitant continuous infusion of epirubicin (an anthracycline drug) 30 $\mathrm{mg} / \mathrm{m}^{2} /$ day for three days. The schedule was repeated every four weeks. The patient's severe pain (9 on a 10-point visual analogue scale) subsided dramatically by day seven after starting chemotherapy. The maximal response was a partial response, which was achieved after four courses. There were no dose reductions or treatment interruptions and granulocyte colony-stimulating factor was administered for support. The maximal toxicities included grade 4 neutropaenia and grade 2 anaemia and thrombocytopenia. The nonhaemotologic toxicities included grade 2 vomiting and anorexia. Her progression-free survival was approximately two months. Salvage surgery (tumour resection) at the time of disease progression was performed at two months after the sixth course of chemotherapy. Follow-up remained uneventful until June 2005, 10 months after starting chemotherapy. She reported gradually aggravating dyspnoea and right flank pain. The CT scans disclosed recurrent effusion and a metastatic tumour over the right psoas muscle. She received further salvage chemotherapy including paclitaxel and cisplatin and concurrent radiotherapy. The patient did not respond to the salvage therapy and died in November 2005, approximately 16 months after the initial chemotherapy was administered.

\section{DISCUSSION}

This case report describes a young woman whose breast tumour was diagnosed as angiosarcoma after sonography and mammography and excisional biopsy. She was treated with rescue chemotherapy using a combination of high-dose tamoxifen plus ifosfamide and epirubicin (an anthracycline). She achieved a partial response, and died 16 months after therapy was initiated.

A study involving 300 women diagnosed with soft tissue sarcomas of the breast was conducted previously (3). Of these, 55 had angiosarcomas, and 32 were primary tumours. The median overall survival for all patients was 2.96 years. The five-year overall survival rates for the 32 patients with primary presentation of angiosarcoma of the breast were $59 \%$, with a median overall survival for this group of 6.53 years. Among those 55 patients, 37 patients were treated with anthracycline-based regimens alone or in combination with ifosfamide or paclitaxel. No data in this 
report specifically showed the medium year of survival or survival rate of the patients receiving chemotherapy (3). Another report showed that the five-year disease-free survival was an estimated $76 \%$ for patients with grade 1 tumours, and only $15 \%$ for patients with grade 3 tumours (4). In the study reported by Scow et al, five-year survival was only $46 \%$ and median time for diagnosis to death was 2.3 years (range 1-13.5 years) for patients with primary angiosarcoma (6). Those patients were treated with mastectomy followed by chemotherapy, radiotherapy, or both (6).

Similar to the previously cited reports, the woman described in this report was young at the time of diagnosis (24 years). Clinical signs were consistent with previous reports [ie palpable mass, skin colour changes] (2). She refused treatment after the initial diagnosis. Four years later, she underwent combined chemotherapy at an advanced stage of disease. She survived for an additional 16 months. Thus, time from diagnosis to death was over five years, which is longer than the median time from diagnosis to death reported by Scow et al (6).

To date, there is no clear consensus regarding optimal treatment for primary angiosarcoma of the breast, but chemotherapy is the primary treatment modality for metastatic soft tissue sarcomas (7). Doxorubicin (an anthracycline antibiotic) either alone or in combination with ifosfamide or dacarbazine, has been a mainstay since the 1970s.

According to Scow et al, responses of soft-tissue sarcomas to doxorubicin-based protocols are approximately $25-35 \%$. Overall survival does not improve with combination drugs versus single agent doxorubicin (6). Ifosfamide has been used successfully for a variety of sarcomas; however, the efficacy of ifosfamide for angiosarcoma remains unclear. More recently, the merits of paclitaxel for angiosarcomas have been noted $(6,7)$. Paclitaxel was not selected as the initial agent for the patient described herein since this drug was not reimbursed by the Taiwan National Health Insurance. In this case, combination was selected based on the report by Yeh et al (8). They described that a good partial response with nearly total tumour reduction in metastatic uterine leiomyosarcoma was achieved by simple addition of high-dose tamoxifen into a previously inactive ADI regimen [adriamycin, dacarbazine and ifosfamide] (8).

\section{CONCLUSION}

In this case, a patient with advanced disease was treated with a combination of high-dose tamoxifen plus ifosfamide and epirubicin (an anthracycline). This patient was treated long after the initial diagnosis but she still achieved a partial response and survived for an additional 16 months. More research is needed to devise novel chemotherapeutics and protocols to improve outcomes in women diagnosed with primary angiosarcomas of the breast.

Author's note: All authors declare no conflict of interest. This work was not supported by any grants.

\section{REFERENCES}

1. National Cancer Institute. Surveillance Epidemiology and End Results Program. SEER Stat Fact Sheets: breast cancer. [cited 2011 Dec 19]. Available from: http://seer.cancer.gov/statfacts/html/breast.html

2. Bae SY, Choi MY, Cho DH, Lee JE, Nam SJ, Yang JH. Large clinical experience of primary angiosarcoma of the breast in a single Korean medical institute. World J Surg 2011; 35: 2417-21.

3. Vorburger SA, Xing Y, Hunt K, Lakin GE, Benjamin RS, Feig BW et al. Angiosarcoma of the breast. Cancer 2005; 104: 2682-8.

4. Glazebrook KN, Magut MJ, Reynolds C. Angiosarcoma of the breast. AJR Am J Roentgenol 2008; 190: 533-8.

5. National Comprehensive Cancer Network. NCCN Clinical Practice Guidelines in Oncology. Breast cancer. V.1.2010. [cited 2011 Dec 19]. Available from: http://img.medscape.com/article/715/307/BreastV.1_ 2010 (Medscape).pdf

6. Scow JS, Reynolds CA, Degnim AC, Petersen IA, Jakub JW, Boughey JC. Primary and secondary angiosarcoma of the breast: the Mayo Clinic experience. J Surg Oncol 2010; 101: 401-7.

7. Movva S, Verschraegen C. Systemic management strategies for metastatic soft tissue sarcoma. Drugs 2011; 71: 2115-29.

8. Yeh KH, Lu YS, Hsiao CH, Cheng AL. High-dose tamoxifen modulates drug resistance to doxorubicin, dacarbazine and ifosfamide in metastatic uterine leiomyosarcoma. Anticancer Res 2003; 23: 5133-7. 